\title{
Hacia un plan nacional de urbanismo. Su necesidad, significación y posibilidades.
}

La concepción general de los problemas del Urbanismo ha variado profundamente en los últimos decenios. El Urbanismo ha consistido durante muchos años en el arte de hacer un trazado más o menos brillante y en llevar estas trazas a la práctica abriendo grandes avenidas, y disponiendo los necesarios servicios. Evidentemente, toda esto ha sido, es y seguirá siendo Urbanismo; pero hoy en día esta labor no es la única propia del Urbanismo, y creo poider decir también que ni siquiera es la más importante. El concepto se ha ampliado mucho, habiéndose complicado por una parte y extendido enormemente en cuanto a su jurisdiccióu territorial.

En cuanto a la naturaleza del problema se ha pasado de considerar la reforma o la ampliación de una ciudad, no ya como cuestión simplemente de forma de trazado, sino como análisis de la manera de ser la organización íntima de la ciudad como entidad social, para poder darle una conformación material que esté en relación lógica y en armonía con las necesidades, las funciones y la idiosincrasia de la ciudad como ser social. Es decir, que antes de proceder al proyecto de unas calles, de unos edificios o de cualquier servicio, es necesario proceder a un análisis sistemático que nos permita conocer cuál es el cuadro funcional en que se mueve cada ciudad, establecer el diagnóstico de los defectos y conflictos de que adolece y abrir cauce a una futura ordenación mediante el señalamiento de un programa de actuación.

En cuanto el problema se plantea así es evidente que el Urbanismo se convierte en un amplísimo campo de colaboración de toda cla- 
se de actividades: la Sociología y el Derecho, la Economía y las Finanzas, la Sanidad, la Administración, la. Técnica y el Arte; todos los más diversos campos profesionales tienen una intervención, y una intervención que ha de ser activa. Claro está que para cumplir esta misión ha de despertarse una inquietud estimulante que dé lugar a un ambiente cultural y a un eistado de opinión dentro de los cuales el equipo de especialistas pueda actuar de una manera firme y solvente.

Además de las complicaciones de todo orden que surgen de este planteamiento y en relación íntima con el mismo, el Urbanismo ha extendido su campo de acción de tal manera que su materia ya no es. solamente la ciudad como congregación urbana de una cierta densidad, sino que cada vez más hay que considerarla con el medio geográfico, social y económico que la circunda. Así resulta que la ordenación de una ciudad hay que estudiarla comprendiendo todo su espacio vital, es decir, teniendo en cuenta los fenómenos humanos que la condicionan, entre los que se destacan los movimientos de emigración, los factores económicos, las circunstancias de su abastecimiento, el hinterland de sus comunicaciones, la naturaleza de su economía y tantos otros aspectos, entre los que han de prevalecer los de índole espiritual, pues no podemos olvidar que las ciudades son cuna y sede de culturas y de toda clase de organizaciones humanas.

Al profundizar en esta trayectoria de la influencia urbana en los ámbitos comarcales y regionales, nos encontramos con que muchas ciudades y comarcas tienen funciones nacionales y que sus actividades y su porvenir están estrechamente ligados a problemas de conjunto, de manera que circunstancias que parecen de índole local, no son más que la consecuencia de premisas nacionales, cuya solución está totalmente fuera del ámbito local. Resulta, pues, señores, que sin plantear el problema urbanístico en su intima naturaleza, como necesariamente hay que hacerlo, y siguiendo una concatenación lógica de las ideas, nos encontramos con que no será posible decir la última palabra de la ordenación de nuestras ciudades, sin tener en cuenta un punto de vista nacional.

Claro está que siguiendo este orden de ideas no tendríamos por qué pararnos en el estrato de lo nacional, sinoi que podríamos perfectamente continuar la ampliación del campo a través de las zonais estratégicas, los continentes y la unidad universal. Es el camino que lleva el mundo y no es fácil predecir hasta qué punto esta expansión ordenadora haya de detenerse en alguno de estos niveles o necesa- 
riamente deba culminarse dentro de planos mundiales. El tema que hoy planteamos es el de: posible Plan Nacional y, por lo tanto, en él detenemos nuestra expansión.

Consecuencia inmediata de esta forma de ver las cosas es que al urbanista ya no le interesan solamente las ciudades, sino que ha de plantearse previamente cuál es la mejor manera de establecer una ssolidaridad social para cubrir unos fines nacionales dentra de determinado marco geográfico. Esta solidaridad social, que en lo material se traducirá siempre en materia urbanística, podrá dar lugar a una gran ciudad concentrada en pequeño espacio, podrá ser también una constelación de ciudades y de pueblos o una dispersión de casas y aldeas, o bien una mezcla de todos estos elementos con una fisonomía y una personalidad determinadas. En principio es igualmente materia urbanística la disposición en una comarca de casas aisladas cuya solidaridad se establece a base de centros cívicos, servicios y comunicaciones de muy amplios radios de acción, que la que resultara de concentrar a todos estos mismos habitantes en un pequeño perímetro con sus calles, plazas y servicios comprimidos y apretados hombro con hombro. La organización urbanística en uno y otro caso es en el fondo idéntica y sus leyes generales de organización son las mismas.

La diferencia no es mayor que la que existe entre un ejército encuadrado en un desfile o el mismo ejército dispuesto sobre el campo para unas operaciones. Los hombres, la organización, la jerarquía, los elementos sociales, todo es idéntico, lo único que varía es la postura de todos estos elementos sobre la geografía.

Dentro de la trayectoria que acabo de señalar, vamos a ver qué cuadro presentan en su conjunto: las ciudades y las comarcas españolas.

Guiándonos todavía por el censo de $\mathbf{1 9 4 0}$ nos encontramos con que en España hay dos grandes ciudades que sobrepasan el millón de habitantes, 16 ciudades que exceden de $\mathbf{1 0 0 . 0 0 0}$ habitantes sin llegar al millón, 20 comprendidas entre 50 y 100.000 habitantes y 95 entre 20 y 50.000 .

Totalizando su población para ver lo que representan en el conjunto de la nación nos encontramos con que las ciudades de más de 100.000 habitantes suponen $\mathbf{4 . 7 0 0 . 0 0 0}$ habitantes; el grupo entre 20 y $100.000,4.100 .000$ habitantes, en tanto que los núcleos inferiores representan de 5 a $20.000,5.000 .000$; de 1.000 a $5.000,6.500 .000$, y los núcleos inferiores a 1.000 suman 2.200 .0000 . En porcentaje sobre la población nacional estas cifras suponen, la primera, el 18 por $\mathbf{1 0 0 ,}$ 
y las siguientes el 16 por 101), el 31 por 100 , ell 25100 y el 10 por 100 . Si lais presentamos en tres grupos nos encontramos con que el conjunto de poblaciones de más de $\mathbf{2 0 . 0 0 0}$ habitantes cuentan con el $\mathbf{3 5}$ por 100 de la población española, en tanto que las poblaciones inferiores a 5.000 habitantes suponen el 34 por 100, y entre ambos grupos en el centro el núcleo de poblaciones entre 5 y 20.000 habitantes contienen el $\mathbf{B 1}$ por $\mathbf{1 0 0 .}$

Claro está que estas cifras tan simples sólo sirven para dar una primera aproximación sobre el reparto de la población en el marco nacional. La Estadística tiene que utilizar una serie de sumandos que se derivan de la situación administrativa y que muchas veces no representan la verdad de la referida distribución geográfica de la población. Así sucede que poblaciones que constituyen una unidad urbanística perfectamente definida, como el Gran Bilbao, con 350.000 habitantes, figuran en el censo subdivididas con 195.000 habitantes para Bilbao y el resto de la población repartida entre distintos grupos. En cambio otras ciudades como Murcia, que en el censo figura con $\mathbf{1 9 3 . 0 0 0}$ habitantes, no contiene en el casco de la población más que $\mathbf{6 0 . 0 0 0}$ habitantes, y el resto es población repartida en un término municipal muy amplio que abarca todos los pueblos rurales de la vega. Cada ciudad y cada comarca tienen en este sentido una fisonomía y una manera de agrupar y organizar la población peculiares, y en esto, como en tantas otras cosas, se acusa la personalidad y la extraordinaria variedad de nuestras regiones y de cada una de las provincias y ciudades. Mientras que en algunas comarcas la población tiende a distribuirse a base de una diseminación casi total de la población, situando la vivienda junto a la parcela de trabajo, en otras toda la población rural se concentra en núcleos no inferiores a $\mathbf{1 0 . 0 0 0}$ habitantes. Entre soluciones tan extremas hay una gama de posibilidades de agrupación que ccmprende las pequeñas condensaciones de 50 a 200 habitantes diseminadas, la ordenación de aldeas de 200 a $\mathbf{1 . 0 0 0}$ habitantes y la concentración de la población en pueblos pequeños comprendidos entre $\mathbf{1 . 0 0 0}$ y 10.000 habitantes. Estos tipos de aglomeración tampoco se presentan en la mayor parte de los casos en sus formas puras, sino que se asocian entre sí dando lugar a soluciones peculiares derivadas de las circunstancias geográficas, económicas y humanas de cada caso. Así, por ejemplo, en Guipúzcoa se da la fórmula muy equilibrada de distribuir la población en tres partes: una tercera parte concentrada en una capital de carácter administrativo y turístico con elevado nivel 
de vida ; ctra tercera parte repartida enrte unos 25 pueblos de alrededor de $\mathbf{5} .000$ habitantes, que en un medio ya urbano, pero sin perder contacto con el campo, contiene la mayor parte de la industria de la provincia; quedando el otro tercio diseminado por los montes, en caserios compietamente ligados a la explotación rural. Pues bien, las diferentes regiones y comarcas ofrecen numerosas simbiosis cuyas características deben conocerse y tratar de que se conserven o que evolucionen en razón de nuevas circunstancias que se vayan presentando. En términos generales es conocido que las diseminaciones corresponden a las zonas cantábricas y a los cultivos de huerta, que las aldeas y los pequeños pueblos corresponden a la meseta, y que las concentráciones mayores se establecen en el Sur y en el Mediterráneo. Se dibuja también con claridad una línea que va de Cáceres a Alicante que separa la zona de los núcleos de población muy numerosos y muy reducidos, de la zona de características contrarias, es decir, de menos núcleos de población, pero con mayor número de habitantes. Sería del mayor interés contar con un criterio bien definido sobre las ventajas e inconvenientes de estas diversas maneras de colonizar previo un análisis detenido de las razones de tipo económico, social, político y militar que las han originado, y un examen desapasionado de las ventajas e inconvenientes que se están derivando de estos hechos. Este estudio, que encierra en sí mismo enormes sugestiones, resulta además hoy imprescindible ante la realidad fundamental de nuestos tiempos, constituída por el crecimiento intenso y continuo de la población.

Si en las últimas generaciones el problema social pudo ser el de tantear las fórmulas modernas de convivenciä, hoy en España el problema esencial que tenemos de cara, es el de buscar con decisión los moldes de la España del próximo futuro en el que sobre el solar español van a convivir alrededor de $\mathbf{4 0}$ ó 50 millones de habitantes. La España de 1900 con 18 millones de habitantes y la España del año 2000 con los 45 millones, que pueden alcanzarse si el crecimiento demográfico se sostiene al ritmo: operante desde 1910, han de ser completamente distintas.

¿Cómo concebimos la cistribución sobre nuestro territorio de estos 45 millones de españoles? Sobre todo, ¿dónde se van a situar los 18 millones que pueden preverse de aumento en este medio siglo próximo? Desde puntos de vista diferentes del urbanismo, pero que le condicionan extraordinariamente, habria que preguntar también: ¿de qué van a comer, en qué van a trabajar y cómo vamos a 
poder elevar el nivel económico de los actuales y futuros habitantes, para poder obtener una estabilidad social al margen de continuas convulsiones?

Continuamente se oye clamar contra las grandes aglomeraciones y contra la emigración de las gentes del campo a la ciudad y, de vez en cuando, Gobernadores y Alcaldes, insinúan la solución para las grandes capitales de nc permitir la inmigración, devolviendo las gentes a su lugar de origen. Claro está que de nada sirven estos clamores y estos propósitos frente a unas leyes naturales producidas inexorablemente por el instirito de conservación. Ocurre que estamos ante un problema impresionante frente al cual nos hallamos, al menos de momento, impotentes para abordarlo. El crecimiento nacional antes señalado de aumento de nuestros habitantes en un 10 por 100 cada diez años, da como resultado que el excedente del medio rural donde el nivel de vida es mínimo y donde la miseria no puede compartirse con más gente, tenga que acudir forzosamente a los puntos de la nación donde exista una posibilidad de encontrar trabajo, en virtud de las nuevas colonizaciones, del programa de industrialización, y en último término, de los servicios que se originan en lass grandes aglomeraciones.

Sucede normalmente que una marea continua de emigrantes va colmando todas las ciudades y comarcas que ofrecen perspectivas de porvenir. La idea simplista de que las gentes acuden del campo a las ciudades por el resplandor de los atractivos y de los vicios que en ellas prosperan, que en sí tiene naturalmente una cierta realidad, conduce, sin embargo, a conclusiones completamente falsas si se piensa que es el motor fundamental por el que las grandes ciudades, al menos en España, crecen tan intensamente. El problema es mucho más grave, es el de subsistir, como sea y donde sea, en el campo, en el pueblo, o en el suburbio. Cuando las gentes encuentran comarcas campesinas de colonización creciente que les permite el asentamiento, acuden a ellas con la misma insistencia con que van a las ciudades, pero naturalmente toda colonización requiere la inversión de capitales como base previa para una posibilidad de inmigración. Esta afirmación se comprueba en España sin más que observar la continua afluencia de población a las cuencas del Guadiana y del Guadalquivir, en donde el crecimiento es continuo e intenso desde hace un siglo. Así, la provincia de Ciudad Real, sin núcleos urbanos de importancia, ha crecido desde 1900 hasta 1940 un 65 por 100, mientras que la totalidad de la nación sólo ha crecido el 39 
por 100, y este crecimiento es la continuación del que tuvo lugar a lo largo de todo el sigio pasado, pues desde 1787 hasta 1900 la provincia de Ciudad Real creció un 135 por 100, mientras que la nación entera creció el 78 por 100. Algo semejante puede decirse de Jaén, Córdoba, Sevilla y Badajoz.

Desde hace siglo y medio existen unas corrientes naturales de movimiento de población dentro de España, que con una regularidad comparable a la de las leyes hidráulicas van embalsando población en comarcas, pueblos y ciudades y resulta imposible torcer estas corrientes si previamente no se han construido los nuevos cauces hacia donde la población pueda orientarse, y así se llega a un dilema que requiere bien amplia meditación.

Si estas corrientes naturales se siguen produciendo como hasta ahora de aquí a fin de siglo: un conjunto de ciudades que sobrepasa ampliamente el número de las capitales seguirá creciendo con un ritmo doble que el del crecimiento nacional, es decir, crecerá el 20 por 100 cada diez años, la que supone que duplican su población en cuarenta años y la triplican en sesenta, en tanto que una serie de comarcas y villas recibe un crecimiento medio del 15 por 100 y el resto de la nación queda estacionario. El resultado será que el año 2000 Madrid y Barcelona tendrán de 3 a 4 millones de habitantes cada una, que Valencia se acercará a los dos millones, Sevilla y Bilbao tendrán alrededor de un millón, otras 20 poblaciones habrán pasado de los 200.000 habitantes y habrá un total de unas 60 poblaciones de más de $\mathbf{1 0 0 . 0 0 0}$ habitantes.

Observando la totalidad de la población española y considerándola dividida en los tres mismos grupos a que antes nois hemos referido de núcleos hasta 5.000 habitantes, de 5 a 20.000 y de má; de 20.000, nos encontramos con que mientras en el año 1900 las pr:porciones respectivas entre los tres grupos eran de 51, 28 y 21 por 100 , en 1940 son del 34, 31 y 35 por 100 , y en el año 2000 serán de' 20,30 y 50 por 100 . Es decir, que la población rural habrá pasado de ser la mitad en 1900 a la tercera parte en 1940, y a la quinta parte en el año 2000. La población de las villas de 5 a 20.000 habitantes se habrá sostenido en porcentajes alrededor del 30 por 100 y la po. L!ación urbana se habrá incrementado en proporción inversa a la disminución de la población rural, pasando del 21 por 100 en 1900 al 35 por 100 en 1940 y a, por lo menos, el 50 por 100 en el año 2009. Así, la pob'ación urbana, que en 1900 suponía 4 millones escasamente, 
y que en 1940 es de unos 8.800 .000 habitantes, alcanzará en el año 2000 , por lo menos, a 23 millones de habitantes.

$¿$ Cabe hacer algo: por variar este porvenir? ¿Es conveniente prever una actuación dirigida hacia otra distribución de la población? En la contestación a estas preguntas radica el fundamento del Plan Nacional de Urbanismo.

En principio y con objeta de trillar un poco el campo tan amplio que ha de abarcar una contestación a estas preguntas, creo que podría sentarse de antemano alguna afirmación previa que nos sirviera de orientación.

Sin duda el crecimiento tan fuerte de las ciudades. nos resulta agobiante y se presenta nuestra economía con solución deficitaria, pues las ciudades no han asimilado ordenadamente tan fuerte desarrollo, y así, los problemas de suburbios, transportes y anarquía general de construcciones están al día en todas nuestras ciudades. En este sentido el retrasar en lo posible el crecimiento de las mayores ciudades y favorecer, en cambio, el desarrollo de los pueblos y ciudades. pequeñas, es decir, de las entidades de población que ofrecen las ventajas esenciales de la vida urbana, sin perder un contacto fácil y natural con el medioj rural, sería sin duda un beneficio social claro.

De este criterio se derivaría una cierta protección a los pueblos de 5 a 20.000 habitantes y a las pequeñas ciudades cuyo volumen podemos señalar entre 50 y 100.000 habitantes.

Por otra parte, creo que también se puede afirmarj que dada la escala de la vida moderna y los medios de mecanización existentes, el problema técnico de las ciudades tiene una solución perfectamente armónica, y que el límite superior de una aglomeración de perfecto funcionamiento debe situarse en una concentración bastante alta.

Los Planes de Madrid y Barcelona para 3 ó 4 millones de habitantes son perfectamente dominables en el orden técnico, y en ellos es perfectamente compatible el rendimiento colectivo y la defensa de los valores humanos siempre que la ordenación responda a una concepción orgánica, de tal manera que los centros representativos y comerciales, los barrios residenciales, las zonas industriales y los poblados satélites constituyan un conjunto en el que las partes tengan personalidad y autonomía propias, y se conserven los vínculos elementales de vecindad y asociación en el trabajo que constituyen los estadios más elementales de una convivencia consistente y perdurable. La dificultad estriba en lo precario de nuestrols medios económicos y en la falta de preparación general para abordar las soluciones 
adecuadas a estos temas, y a defecto de una buena ordenación, si es cíaro que una aglomeración de 4 millones de habitantes inorgánica da lugar a un medio social de graves peligros. Si esto decimos de las más grandes ciudades es fácil deducir que para lass de 100 a 500.000 habitantes los problemas son semejantes con un margen menor de dificultades; su solución técnica es más abordable y los riesgos de desorden menores.

De todo esto se deduce que por una parte conviene proteger por consideraciones económicas y sociales los pueblos de tipo medio y las pequeñas ciudades y que por otra parte tampoco hay que asustarse demasiado de las grandes ciudades siempre que contemos con un mecanismo adecuado para organizar su crecimiento y de los medios financieros para edificarlas dignamente.

En el orden práctico resultaría un poco bizantino el pronunciarse, (a priori), por soluciones de uno $u$ otro tipo, porque el verdadero problema está en discernir la especie de simbiosis más adecuada para combinar unos y otros tipos de población y de collonización, para obtener una situación armónica en relación con la geografía, la economía y con los fines de todo orden de la nación. Todos los tipos diversos integrantes de la población española son en principio buenos si corresponden a. una estructura de conjunto racional y eficiente. E] daño llegará si se produce el desequilibrio entre las diferentes partes integrantes. Así, por ejemplo, un gran desarrollo urbano que no fuera seguido de una fuerte industrialización y que se practicara de espaldas al campo sería una fuente de toda clase de males y desórdenes. Los tres programas esenciales del trabajo nacional que pudiéramos resumir en agricultura, industria y servicios urbanos, tienen que tener una dimensión y un crecimiento que guarden entre sí relaciones determinadas. De aquí resulta que una concepción nacional del futuro urbanístico no puede esbozarse si no se tiene al mismo tiempo una idea concreta de cómo va a progresar la agricultura y el abastecimiento nacional por una parte y la industria y la elevación de nivel, de vida por otra.

El hecho de esta interconexión entre temas y problemals tan complejos, hace que sea muy difícil trazar un esquema que pueda servir de guía a un planeamiento nacional urbanístico. Pero por otra parte no hay otro camino para llegar a la visión conjunta que esbozar desde cada campo todo el horizonte mayor posible. Pues bien, si quisiéramcis interferir en las corrientes naturales de población de que antes hablaba y orientarlas hacia formas de acuerdo con premisas 
establecidas, tendríamos que actuar en las fuentes económicas. Habríamos de saber dónde y en qué cuantía puede intensificarise la producción agrícola y asimismo tendríamos que establecer la hipótesis adecuada de localización de todo el programa industrial a desarrollar en cincuenta años. De esto:s programas iniciales deduciríamos los correspondientes a las comunicaciones y al comercio y por último concretaríamos un programa de construcciones y servicios típicamente urbanísticos, que comprendería desde las viviendas hasta los centros directivos y representativos. Para no proceder quiméricamente tendríamos que conocer, lo mismo que hacemos para el movimiento de población, las corrientes económicas existentes y su distribución en diferentes ramas y a través de las diversas regiones. Lo mismo que se examinaba el incremento de población y su localización a través de los decenios, hay que realizar el estudio de los incrementos de riqueza y de su localización.

En este nuevo orden de ideas nos encontramos con que también la riqueza se distribuye en formas muy diferentes: en unas regiones se concentra en pocas manos, y en otras se desparrama en sectores más amplios y en general en cada una hay una fisonomía determinada de asociación de tipos determinados de volumen de riqueza. De una manera paralela a lo que sucede a la población, la riqueza es también estable en unas regiones y creciente en otras, y los tipos de asociación son unas veces favorables para la convivencia pacífica y pueden ser en otros casos fuente de graves perturbaciones sociales. Una ordenación urbanística adecuada de la población debería ser en su crecimiento paralela a los incrementos de riqueza, de tal manera que donde no hubiera posibilidad de ahorro no existiera tampoco crecimiento de población, y que éste acudiera en mayor o menor intensidad a las ciudades y comarcais según sus posibilidades de incremento de riqueza. Esto en la práctica sucede unas veces sí y otras no. En este sentido el desequilibrio urbanístico español es muy grande. Vamos a verlo.

España ofrece cuatro centros de inmigración fundamentales, que son: Madrid, Barcelona, las Provincials Vascas y las cuencas del Guadalquivir y Guadiana; pues bien, su fisonomía económica, en relación con el urbanismo, es bien diferente. Las Provincias Vascas crecen a base de su desarrollo industrial que produce un ahorro que permite atender al crecimiento urbanístico, y éste se lleva a efecto en una ciudad importante, pero no excesiva, como Bilbao; una ciudad equilibrada, como San Sebastián, y un conjunto de ciudades me- 
nores y pueblos importantes que dan lugar a una distribución de la población y de la riqueza con un cierto equilibrio, quedando además margen para que una parte del capital vasco se emplee en obras de colonización y urbanización en otras regiones españolas.

En Cataluña, con una agricultura excepcional y una industria floreciente, la mayor parte del incremento de población y del ahorro se invierte en la ciudad de Barcelona, que de esta manera compite urbanísticamente con Madrid; en cambio el resto de las capitales provinciales y la mayor parte de los pueblos isufren un abandono grandísimo, de tal manera que a pesar de contar con elementos de riqueza relativamente abundantes, su nivel urbanístico: es inferior, por ejemplo, al de las ciudades del Norte.

En los valles del Guadalquvir y el Guadiana el incremento corresponde a una colonización cada vez más intensa, pero al no existir una tradición industrial, el ahorro del campo se invierte en el campo. y no existen capitales para construir ciudades, y así nos encontramos con que las ciudades andaluzas, pongo por ejemplo, Sevilla, Granada, Málaga y Córdoba, han crecido como las que más, sin realizar un ensanche que valga la pena. La población se aglomera en los cascos antiguos, convierten los palacios en casas de vecindad y crean míserols suburbios en sus alrededores. De la misma manera que han triplicado su población últimamente lo habrán de hacer en el próximo futuro, y no se ve de dónde pueden salir los capitales y cómo puede surgir la industria que permita vislumbrar un porvenir razonable.

Madrid, como capital de España, participa de la inmigración general de gentes y ahorros. Al no contar con una industria propia suficiente, depende de los excedentes de la nación.

$\mathrm{He}$ aquí cuatro estampas bien distintas del panorama nacional cuyo cuadro total no sería difícil completar examinando las características del NO., de las regiones centrales, de Levante y de las Islas. En términos generales nos encontramos con una cierta autonomía económica y urbanística en Levante y en las Islas y con un proceso retrasado en ambos sentidos en los otros dos sectores.

Un Plan Nacional de Urbanismo tendrá que fundamentarse en el conocimiento preciso de las fuentes del ahorro español, de su distribución entre las diferentes necesidades y de su posible localización.

El único procedimiento práctico para influir en las corrientes de distribución de población será realizar una adecuada política econó- 
mica que oriente las inversiones hacia los fines más convenientes y hacia las localizaciones que puedan interesar desde el punto de vista nacional. Las mayores o menores posibilidades de intervención en el desarrollo: de todos estos fenómenos están condiciomados por lo tanto a las posibilidades existentes de modificar las inversioness tradicionales. Si procedemos a la inversa, habrá que decir también que no se puede realizar una política económica nacional sin tener en cuenta los problemas tan esenciales que se derivan de un planteamiento urbanístico nacional.

Cuando oímos que el crecimiento de Madrid, de Barcelona o de cualquiera de las otras ciudades españolas debe ser deitenido, debemos saber que eso significa la indulstrialización de Galicia, la elevación del nivel de vida de Castilla o la colonización a fondo de Extremadura y Andalucía, y que no se puede pensar en impedir el crecimiento de población gozando, sin embargo, de los incrementos de riqueza. ¡Eso sí que sería el máximo desequilibrio nacional! En elsta forma tendríamos un pequeño grupo de capitales con alto nivel de vida en medio de una España empobrecida y hambrienta.

Con todo esto creo que queda patiente la dimensión del problema y la necesidad de conocerlo a fondo y de establecer unas bases scbre las que poco a poco pueda levantarse el andamiaje de disposiciones, planes y colaboraciones indispensables para poder llevar a la práctica una labor de este género:

Comoj todos saben, otros países se nos han adelantado en esta actuación y cuentan ya con organismos ejecutivos que trabajan por estos isenderos. Buen ejemplo es el Ministerio inglés de Planeamiento, que últimamente ha cambiado de nombre denominándose de Urbanismo y Administración Local. En Francia cumplie un papel análogo el Ministerio de la Reconstrucción. Entre nosotros la Dirección General de Arquitectura surgió en 1939 a impulso de un deseo die ordenación del Urbanismo Nacional, ya que resultaba patente el fracaso de los Ayuntamientos por el incumplimiento casi total de las obligaciones impuestas por el Estatuto Municipal. A los diez años, en 1949, la labor ya realizada justifica la creación, dentro de la Dirección de la Jefatura Nacional de Urbanismo, dedicada a impulsar la trayectoria urbanística de la nación, y a la que se asigna entre otras facultades la de establecer los estudios preliminares y colaboraciones necesarias para la preparación de un Plan Nacional de Urbanismo.

Es manifiesta la desproporción existente entre el Organo creado 
y la ingente tarea a realizar, pero es natural que ésta se verifique por grados y que antes de constituir un Organismo de más amplias ambiciones se vayan cubriendo en etapas intermedias los trabajos preliminares. Estos han de ser: la preparación de equipos adecuados, la creación de una mentalidad nueva que permita lass más extensas colaboraciones en todos los campos profesionales, hacia una labor conjunta, la formación de un ambiente favorable en la opinión pública, la obtención de una experiencia suficiente para apoyar en ella actuaciones de gran responsabilidad y por último la iniciación de los primeras pasos sobre los que puedan conjugarse voluntades y esfuerzos.

Siguiendo este camino, sabiendo que con ello se da cumplimiento a una consigna frecuentemente expresada por el Jefe del Estado y con el apoyo constante del Ministra de la Gobernación, se han formado por Ley tres Organismos urbanístico:s de nuevo cuño para la ordenación urbana de Madrid, Bilbao y Valencia, y se han constituído por Decreto 21 Comisiones Provinciales de Ordenación Urbana con la misión de redactar los planes provinciales de Urbanismo. De esta manera, al mismo tiempo que se resuelve un problema tan urgente e inmediato como el de que las ciudades y comarcas crecientes cuenten con sus planes de ordenación, se da un paso importante en el camino de la colaboración, ya que en cada Comisión trabajan, bajo la presidencia del Gobernador Civil, las autoridades locales, Ios representantes de los Ministerios de Obras Públicas, Industria, Agricultura, etc., y los técnicos especialistas, Arquitectos e Ingenieros.

Se contribuye también a la unificación de pareceres y al establecimiento de criterios que puedan ser la base de la propaganda, y se obtiene una base de experiencia y conocimiento para, sobre los resultados de los planes provinciales, poder formar un diagnóstico, cada vez más preciso, de las necesidades urbanísticas nacionales. De esta manera se va perfilando la estructura del dispositivo jerárquico de los Organos Ejecutivos, Asesores y Fiscales que han de desempeñar la misión urbanística y en consecuencia es de esperar que no ha de pasar mucho tiempo sin que tengamos la Ley Orgánica que abra cauce a toda esta tarea.

Las dificultades que se presentan ante nosotros son muy grandes. La evolución del antiguo concepto de actuación dispersa, hacia un frente unificado con posibilidades prácticas de planeamiento cada vez más intensais supone modificar muchos criterios en materias fundamentales. Entre ellas pueden señalarse, por ejemplo: La moviliza- 
ción de las Corporaciones Locales en un sentido de eficacia y responsabilidad, la revisión de las facultades en materia de Urbanismo del Estado y las Corporaciones Locales, la necesidad de romper con los Departamentos estancos en todos los Organismos oficiales. Muy importante es también el planteamiento de una base justa para el desarrollo de una política de suelo evitando las especulaciones abusivas, e incorporando e impulsando las actividades constructivas de la iniciativa privada. Temas todos ellos que sobrepasan grandemente el ámbito de una especalidad o de un Organismo para entrar de lleno dentro de una política de gobierno.

\author{
Pedro Bidagor Lasarte, \\ Arquitecto \\ Jefe Nacional de Urbanismo
}

